\title{
Society of Skeletal Radiology 2008 Annual Meeting, Scientific Presentation Sessions, 9-12 March 2008, La Quinta Resort and Club, La Quinta, CA, USA (Correction)
}

\section{Skelet Radiol (2008) 37:384}

Unfortunately, the names of the authors were given incorrectly in the abstract. Therefore, the abstract is published here again with the correct names of all authors.

The abstracts of the meeting can be found at http://dx.doi.org/10.1007/ s00256-008-0461-4.
33

MRI FEATURES OF POSTERIOR CAPITELLAR IMPACTION INJURIES

Rosenberg ZS; NYU-Hospital for Joint Diseases, New York, NY, USA, Blutreich SI, Schweitzer ME, Zember JS, Fillmore K

Purpose: Posterior capitellar injury is a scantly mentioned entity in the literature. Furthermore, irregularity of the posterior capitellum, noted on MRI and coined the pseudodefect of the capitellum, has been touted as a normal finding, not to be misinterpreted for an impaction injury. The objectives of our study, based on 11 MRI studies, were to 1) Report the MRI features of a true posterior impaction injury of the capitellum and 2) Document associated osseous and soft tissue abnormalities which may shed light onto the etiology of this injury.

Materials and Methods: 781 elbow 1.5T MR examinations performed over 42 months were retrospectively reviewed, via a computer data search, for the presence of marrow edema in the capitellum. All cases with posterior capitellar marrow edema were further assessed for the following: A) The pattern of signal and morphologic alteration of the capitellum, B) The presence of associated collateral ligament injuries and C) The presence of other osseous and soft tissue abnormalities. Review of the medical records was performed in all identified cases of posterior capitellar marrow edema.

Results: A total of $46(5.9 \%)$ cases of capitellar marrow edema were identified. Eleven (24\%) of these (8 males, 3 females, age range 21-94, mean age 42) were in a posterior location. 5 patients described falls as the cause for their injury. There was clinical documentation of elbow dislocation $(n=2)$, posterolateral rotatory instability $(n=4)$, lateral epicondylitis $(n=2)$, and elbow pain $(n=3)$.

The pattern of posterior capitellar marrow abnormalities were diffuse, bone bruise $(n=7)$, subchondral arc like $(n=2)$, subchondral cyst $(n=1)$ and subchondral linear signal $(n=1)$. The capitellar changes were associated with radial head bruises $(n=4)$, radial head fractures $(n=3)$, coronoid process bruises $(n=4)$, and olecranon bone bruise $(n=1)$.

Nine $(82 \%)$ of the 11 posterior capitellar injuries had additional lateral ulnar collateral ligamentous injuries. Five $(55 \%)$ of the 9 cases also had tears of the lateral collateral ligament and $2(22 \%)$ had additional tears of the medial collateral ligament. Other findings included subluxation of the ulnohumeral joint $(\mathrm{n}=1)$ and partial tearing of the common extensor tendon $(\mathrm{n}=2)$.

Conclusion: Traumatic posterior impaction injuries can occur in the capitellum, albeit infrequently. These lesions are often associated with lateral ulnar collateral ligament injury and, less frequently, with lateral and medial collateral ligament injuries. The presence of these lesions, to be distinguished from the pseudodefect of the capitellum, should raise the possibility of a previous elbow dislocation and/or the presence of posterolateral rotatory instability. 